\title{
Research on the Mobile Highway Measurement System Development and Optimization Model with Data Oriented Thinking
}

\author{
Wei Pan \\ Henan Vocational and Technical College of \\ Communications, Zhengzhou city,China, 450000
}

\begin{abstract}
In this paper, we conduct research on mobile highway measurement system development and the optimization model with data oriented thinking. Measurement engineering is one of the oldest subjects which have a long history. It is always with contemporary surveying and mapping science and technology and the development of the project construction phase synchronization. As the space technology, computer technology, communication technology and the development of the geographic information technology, the basic theory of engineering surveying, engineering and technical system, the research and scientific goal is undergoing profound changes. From the surveying and mapping science and technology development in recent years, our country's obvious changes have taken place in engineering survey technology that have also made great achievements. Under this background, we enhance the traditional methods to propose our novel perspective that is meaningful.
\end{abstract}

Keywords- Mobile Highway, Measurement System, Data Oriented, Optimization Model.

\section{Introduction}

Survey of the construction of the modern engineering survey usually refers to the engineering design, construction and management phase in applying theory, method and measurement of the floorboard of the technology engineering survey technology. Definition of the engineering survey: in the design, construction and management of the construction of the project in each stage of the theory and method of the measuring work and technology called engineering survey.

\author{
Lijuan Zhang \\ Henan Vocational and Technical College of \\ Communications, Zhengzhou city,China, 450000
}

Engineering surveying and mapping science and technology is a direct application in national economy and national defense construction, is a comprehensive application of surveying and mapping science and technology, it directly for basic and general engineering construction services [1-3].

Based on the literature review, the separation of the modern measurement system can be listed as the follows. (1) According to basic engineering construction program is classified. According to the construction of belonging that engineering can be divided into the planning and design phase of the measurement, construction phase of the measurement and operation management after completion phase measurement. Planning and design phase of the measurement is mainly provides the terrain data, method of terrain data is established in mapping control survey conducted on the basis of ground or aerial survey. (2) According to the engineering surveying engineering services by primary species classification. According to the engineering surveying engineering services by the types, also can be divided into construction, line measurement, bridge and tunnel, mine surveying, urban measurement and water conservancy engineering measurement, etc. In addition, it will be used in large equipment and high precision degree is known as the high precision engineering surveying and the deformation observation called contemporary photogrammetry technology applied in the engineering construction project photogrammetry, and by electronic total station or ground camera sensors in computer support 
system of measurement called the three-dimensional industrial measurement [4-5].

In actual measurement, the selection of measurement method according to the characteristics of the shape of the object to be tested and application purpose as a kind of basic testing instrument in three coordinates measuring machine in the reverse engineering application development become the main physical appearance digital equipment, measurement speed is slow, but exist the known probe radius compensation is required by measuring the force is too big, can't measure soft faults such as material samples. With continuous development of the hardware technology optical non-contact measurement technology has been developing rapidly, with high response, high resolution. This method has the fast measurement, high accuracy that is not subject to electromagnetic interference environment, working distance, measurable material advantages of soft samples, successfully solved the problem of contact measurement method. In the future development trend, non-contact measurement will be increasingly occupied the main position, but the three coordinate measuring machine still have an advantage in the terms of accuracy. The following figure shows the sample interface.

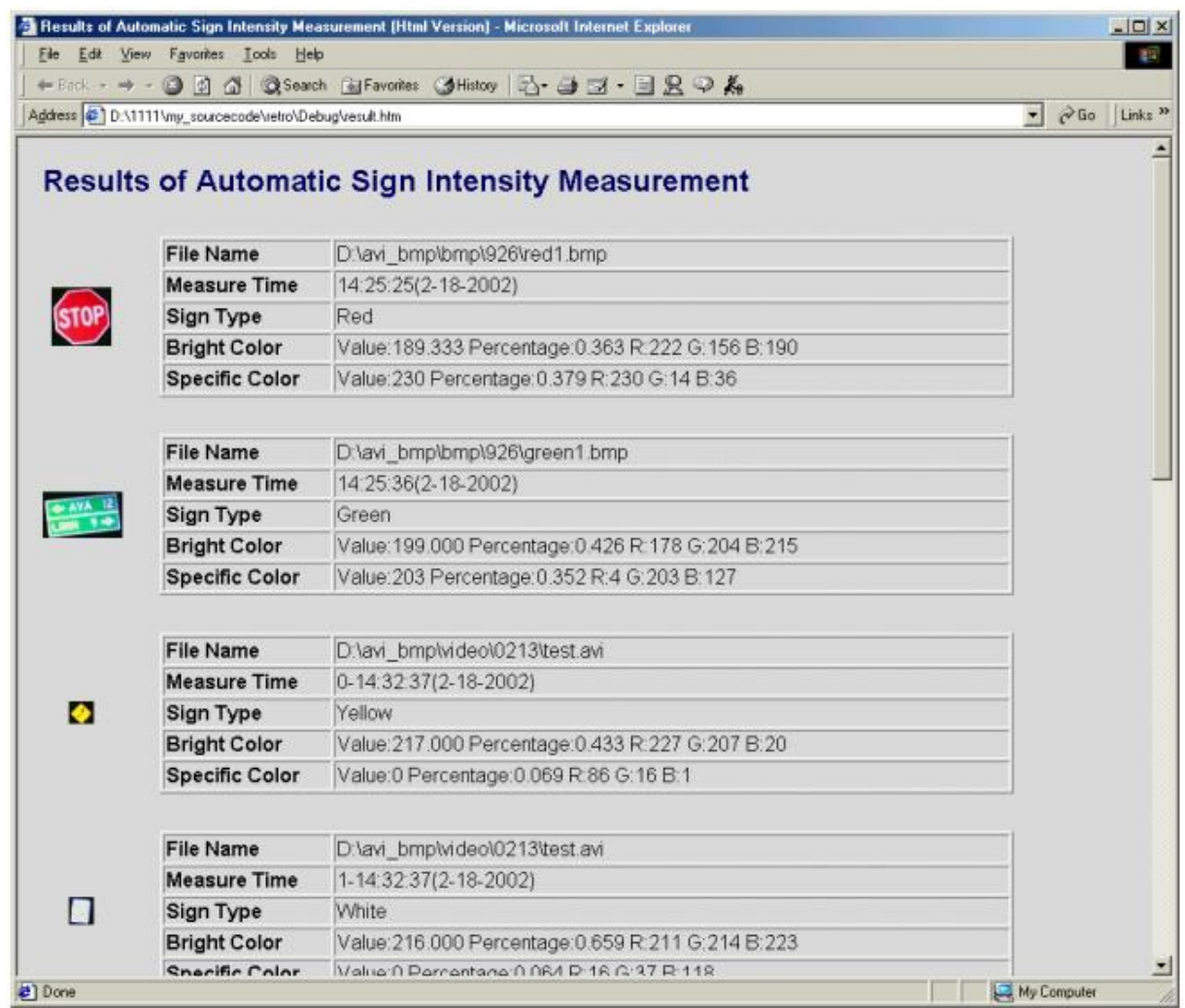

Figure 1. The Sample Interface of the Mobile Highway Measurement System

In this paper, we conduct research on mobile highway measurement system development and the optimization model with data oriented thinking. In the later parts, we will discuss in detail.

\section{The Proposed Methodology}

The Mobile Highway Measurement. As more and more high grade road construction, the difficulty of the route survey work is becoming more and more big, more laying wires, and less known points, using the traditional method of 
construction and it is difficult to net at the same time, it is difficult to complete the high accuracy of the control. Correspondingly, the challenges can be summarized as the follows. (1) Early in preparation for the highway measurement, often due to the density of traverse point set is not reasonable, and will not be able to correct use of gauss plane coordinate measurement, it is easy to appear measurement results and the actual results have large deviation, resulting in the subsequent problems of the highway construction. Calculation results can lead to such as errors in subgrade construction, a super fill or is the issue of overbreak especially those super high wide roads. (2) After the end of the highway engineering construction, the acceptance stage also need of highway measurement, such as to retest of the traverse points and leveling points, but in the process of actual measurement, and no serious measurement. (3) Roadbed is mainly done by primary earth and stone construction of linear structure able to withstand a load weight and road surface gravity and driving. It is one of the most important parts of the constituents of the highway. If the measurement problem in subgrade construction aspects, such as only according to the data line, cross-sectional design ground line error that cannot be combined with the actual terrain to adjust the edge position [6].

For the better measurement performance, we should introduce the corresponding GPS and the data guided models. The application of GPS in highway survey, survey method and operation method of the high-grade highway produced revolutionary change, greatly improve the efficiency of the survey accuracy and surveying, especially the real-time dynamic positioning technology in highway survey, construction and later maintenance, management have broad application prospects. The advantages of GPS measurement could be summarized as follows.

- Measure the radius of broad. The whole line without setting control network, such as basic secondary wires just setting good head control network can complete control work. Recovered as long as the heads of the save is good, can at any time of the whole line or in the midline in the area of the layout work, therefore, we also don't have to worry about some important pile location such as the intersection point of lost and bring difficulties to line measurement.

- Sure height to determine the plane position first. GPS technology can make basic use of 3D coordinate information at the same time determine the elevation of point, the midline of the lofting out the flat measured steps that greatly enhance the efficiency of work.

- Visibility conditions demand is not high. GPS measurements, mainly through electromagnetic wave and GPS satellite signal measurement, without being limited by the topography and the visibility while greatly improving the work efficiency [7].

- Save the human resources. GPS reference station gives the application flow for multiple site location information, the rover one person can complete the operation that greatly saves the manpower, to enhance the resource configuration and improve the efficiency.

At present, using the GPS static or along the rapid static method to establish the overall control measure, surveying and mapping for the survey period banded topographic map, route plane, vertical surface measurements provide basis; In construction stage for the bridge, tunnel construction control network, this is only for the application of the GPS in highway survey primary stage, in fact, highway measurement of technical potential to accumulate in application of RTK technology, the application of RTK technology in highway engineering has a very broad prospect.

The Engineering Surveying. Engineering surveying experienced from simple to complex, from manual operation to the artificial 
intelligence, from contact measurement to non-contact telemetry, from normal to high precision measurement accuracy, from the narrow sense of civil engineering to the generalized engineering measurement of development path. Along with the computer technology, communication technology, space technology and the development of the geographic information technology, engineering survey of the theoretical basis, technical system, research and scientific goals are undergoing profound changes that can be reflected from the figure two.

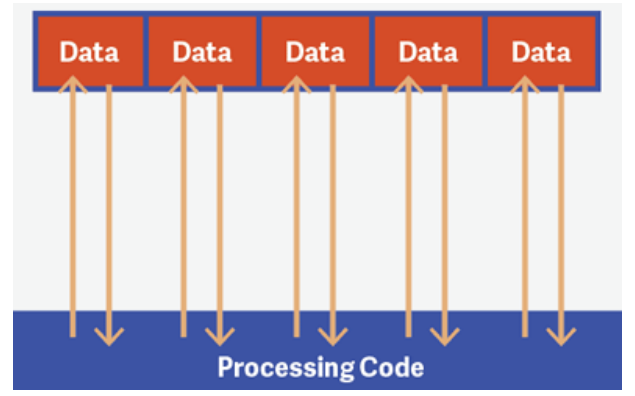

Figure 2. The Data Oriented Paradigm

Due to the progress of science and technology, all kinds of digital technology gradually rise in the engineering survey construction measurement for the application of digital technology has increased. Modern digital measurement technology, it is from the traditional optical and mechanical toward more advanced microelectronics technology and the development of intelligent measurement, the measurement work, can through to the digital measurement machinery to set related to simplify the measuring work, and can provide the accuracy of measurements and informatization related technical support and overall improve the efficiency of the construction engineering surveying work and basic efficiency in the process of construction and as result of measurements in the start stage, construction stage and completion stage has effect, so the digital measurement device can also support depending on the measuring project, equipment parameters for different settings, or select different equipment. And in order to make the equipment more human, modern digital measurement technology related facilities is more portable, integration development. The technology to promote the development of our country construction engineering survey technology played an important role.

Additionally, the modern engineering surveying could be categorized as follows. (1) Engineering surveying positioning. In modern digital measurement technology, digital measurement technology can according to the satellite positioning, through static positioning, use for a long time observation to provide an accurate result, but the downside is that want to accurate results it must cost a lot of time, so for some aren't that strict engineering does not apply, so there is another way of basic fast static positioning. (2) Engineering surveying and mapping. Through the use of advanced technology, reduce the manpower in engineering surveying and mapping it a huge workload to withstand the intensity of labor, and for the quality of the finished product of the surveying and mapping has certain security, through a series of related electronic equipment that has realized the real-time, dynamic positioning, a data acquisition, processing, and various automatic functions such as editing, automatic drawing.

The Measurement System Construction Pattern. Geographic information system applied in the construction of relatively short cycle, in the process of application, the geographic information system can be used to measure all the data needed in the basic engineering project, and also has a very good performance on the accuracy of measurement results. Therefore, in process of building engineering construction, use of geographic information system GIS can better for construction enterprise to bring the huge economic efficiency, for the development of our country construction enterprise also has a very 
positive meaning. For the systematic construction, we list it as the follows.

- The user data processing. In handling of user data, geographic information system GIS can make use of surveying and mapping unit to collect the data of the relevant information, and on the application of system database is also more convenient and quick, different construction projects are different to the requirement of measurement, so the data processing requirements also has the very big difference [8].

- Development needs. Geographic information engineering in construction project construction process can not only provide users with the corresponding core data model, and can also offer corresponding reference to engineering construction, in the process of surveying and mapping, surveying and mapping methods have different requirements, different among more complex information that can be used only after the corresponding processing, and the content of the transformation must ensure that the staff can more clearly understand and understanding, so as to better benefit of construction projects.

- Hierarchical processing. Segmentation is an important feature of the geographic information system, which mainly displays in the system's ability to the data isolation, data positioning function in the field, different data and different content in the process of construction of the function is also different, and the content need to accord with engineering surveying data with high degrees, such ability can guarantee the accuracy of the construction.

- Function module. Powerful data processing function of the system can change measurement information of multiple formats, flexible in construction, system can provide the measurement for different construction management personnel information, such as construction personnel to provide drawings, the engineering design of the surveying and mapping personnel provide geological data, to quality inspection personnel, to provide engineering structure parameters.

\section{Conclusion}

In this paper, we conduct research on the mobile highway measurement system development and the optimization model with data oriented thinking. Engineering survey in the whole process of basic construction engineering construction is a very basic work, the engineering construction plays a very important role, this part once appear problem, to the following the progress of the project will produce very big effect, so the problem, though basis but it is very important. In the actual operation process, engineering survey are influenced by various factors, these factors will directly affect the engineering measurement accuracy, so this paper analyzes these factors, and can be used to its control measures were discussed. Along with the rapid development of the economy, the quality of the project from all parties in society have put forward higher request, so this also indirectly for engineering survey put forward higher request, because it plays a very important role in engineering construction. In future, we will conduct more in-depth analysis for optimization.

\section{Reference}

[1] Stroud, Craig A., et al. "Rapid organic aerosol formation downwind of a highway: Measured and model results from the FEVER study." Journal of Geophysical Research: Atmospheres 119.3 (2014): 1663-1679. 
[2] Pattinson, Woodrow, Ian Longley, and Simon Kingham. "Using mobile monitoring to visualise diurnal variation of traffic pollutants across two near-highway neighbourhoods." Atmospheric Environment 94 (2014): 782-792.

[3] Abbas, Taimoor, et al. "A measurement based shadow fading model for vehicle-to-vehicle network simulations." International Journal of Antennas and Propagation 2015 (2015).

[4] Chevalier, Amelie, et al. "Emulation of a highway bottleneck using leader-follower formation control." Applied Computational Intelligence and Informatics (SACI), 2013 IEEE 8th International Symposium on. IEEE, 2013.

[5] Jalayer, Mohammad, et al. "Comprehensive Assessment of Highway Inventory Data
Collection Methods for Implementing Highway Safety Manual." Transportation Research Board 94th Annual Meeting. No. 15-2288. 2015.

[6] Li, Wenling, and Yingmin Jia. "Location of mobile station with maneuvers using an IMM-based cubature Kalman filter." Industrial Electronics, IEEE Transactions on 59.11 (2012): 4338-4348.

[7] Chowdhury, Mostafa Zaman, et al. "Handover Call Admission Control for Mobile Femtocells with Free-Space Optical and Macrocellular Backbone Networks." arXiv preprint arXiv:1412.4321 (2014).

[8] Song, Xiaoquan, et al. "Design and implementation of vibration isolation system for mobile Doppler wind lidar." Journal of the optical society of Korea 17.1 (2013): 103-108. 